\title{
Die „Domestizierung“ der Parlamente im postkommunistischen Osteuropa: Russland und die Ukraine im Vergleich
}

\author{
Ingmar Bredies
}

M. Steven Fish stellt in dem 2006 erschienenen Aufsatz „Stronger Legislatures, Stronger Democracies“ einen unmittelbaren Zusammenhang zwischen der institutionellen Macht von Parlamenten und den Aussichten auf eine erfolgreiche Demokratisierung in Transformationsstaaten her. ${ }^{1}$ Sein Beitrag knüpft dabei an eine Kerndebatte der vergleichenden Politikwissenschaft an, die von einer Korrelation zwischen dem institutionellen Design von Regierungssystemen und politischen Regimetypen ausgeht. ${ }^{2}$ Parlamentarischen Regierungssystemen wird dabei aufgrund ihrer institutionellen Konfiguration mehrheitlich zugeschrieben, eher die Beschränkung und Verantwortlichkeit politischer Herrschaftsträger gewährleisten zu können sowie breite politische Partizipation und Inklusion zu ermöglichen. ${ }^{3}$ Vor dem Hintergrund der postkommunistischen Systemtransformation scheint der Befund M. Steven Fishs einer ersten empirischen Stichprobe standzuhalten: So verfügt die Mehrheit der nordost-, mittel- und südosteuropäischen Mitgliedstaaten der Europäischen Union über parlamentarische Regierungssysteme - mit entsprechend „starken“ parlamentarischen Vertretungskörperschaften. Die formal als semipräsidentiell zu kennzeichnenden Systeme Litauens, Polens und der Slowakei haben sich tendenziell ebenfalls dem parlamentarischen Grundtyp angenähert. ${ }^{4}$ Da sich im postkommunistischen Osteuropa keine reinen präsidentiellen Regierungssysteme herausgebildet haben, konzentrierte sich die Debatte um die Wechselbeziehungen zwischen der Wahl des Regierungssystems und der Herausbildung neuer Regimetypen zunehmend auf den Semipräsidentialismus, der in der Region zunächst zum „Exportschlager“ avancierte. Diesem Regierungssystemtyp wird jedoch insbesondere für den postsowjetischen Raum eine erhöhte Anfälligkeit für autoritäre Rückfälle attestiert. ${ }^{5}$ In diesem Beitrag sollen einige Grundannahmen über den Zusammenhang zwischen „Parlamentsmacht" 6 und der Herausbildung bestimmter Regimetypen am Beispiel Russ-

1 Vgl. M. Steven Fish, Stronger Legislatures, Stronger Democracies, in: Journal of Democracy, 17. Jg. (2006), H. 1, S. 5 - 20.

2 Dieser Beitrag orientiert sich an der Trias moderner Regimetypen nach Juan J. Linz: Demokratie - Autoritarismus - Totalitarismus.

3 Vgl. Juan J. Linz, The Perils of Presidentialism, in: Journal of Democracy, 1. Jg. (1990), H. 1, S. $51-69$.

4 Grundlegend für eine solche Einschätzung ist in Anlehnung an Winfried Steffani das primäre Kriterium parlamentarischer Regierungssysteme, das in einem „parlamentarischen Misstrauensvotum mit zwingender Rücktrittsfolge“ besteht: ders., Parlamentarisch-präsidentielle „Mischsysteme"? Bemerkungen zum Stand der Forschung in der Politikwissenschaft, in: Otto Luchterhandt (Hrsg.), Neue Regierungssysteme in Osteuropa und der GUS. Probleme der Ausbildung stabiler Machtinstitutionen, Berlin 2002, S. 17 - 65, S. 49.

5 Vgl. Agnes Gilka-Bötzow, „The Perils of Semipresidentialism“? Gewaltenteilung in der Ukraine vor und nach der Verfassungsreform, in: Sabine Kropp / Hans-Joachim Lauth (Hrsg.), Gewaltenteilung und Demokratie. Konzepte und Probleme der „horizontal accountability“ im interregionalen Vergleich, Baden-Baden 2006, S. $261-279$.

6 Zum Begriff der „Parlamentsmacht“ siehe Werner J. Patzelt, Phänomenologie, Konstruktion und Dekonstruktion von Parlamentsmacht, in: ders. (Hrsg.), Parlamente und ihre Macht. Kategorien und Fallbeispiele institutioneller Analyse, Baden-Baden 2005, S. 255 - 302. 
lands und der Ukraine überprüft werden. Dabei wird die Entstehung eines „neuen“ Autoritarismus in Osteuropa - vor allem im postsowjetischen Raum - unmittelbar auf die „Domestizierung "7 von Parlamenten zurückgeführt.

Der Untersuchungszeitraum umfasst die Amtszeiten der russischen Präsidenten Vladimir Putin (2000 bis 2008) und Dmitrij Medvedev (seit 2008) sowie der ukrainischen Staatsoberhäupter Leonid Kučma (1994 bis 2004) und Viktor Juščenko (2005 bis 2010). Ein Vergleich zwischen Russland und der Ukraine bietet sich dabei aus folgenden Überlegungen an: Beide Staaten stehen in den letzten Jahren sinnbildlich für zwei konträre Entwicklungspfade. ${ }^{8}$ Mit der Orangenen Revolution 2004 habe sich einigen Autoren zufolge in der Ukraine ein „demokratischer Durchbruch“9 vollzogen. Zudem trat zum 1. Januar 2006 eine Verfassungsreform in Kraft, die das ukrainische Regierungssystem von einem präsidentiell-parlamentarischen in einen premier-präsidentiellen Subtyp umwandelte. ${ }^{10}$ Die Nichtregierungsorganisation „Freedom House“ wies der Ukraine zudem in den Jahren 2006 bis 2010 als einzigem sowjetischen Nachfolgestaat - mit Ausnahme der baltischen Republiken - den Status „frei" zu. ${ }^{11}$ Der insbesondere in diesen Zeitraum fallenden Demokratisierung durch Parlamentarisierung in der Ukraine steht das politische System in Russland entgegen, dem zahlreiche Politikwissenschaftler eine autoritäre Konsolidierung attestieren. ${ }^{12}$ Korrelieren diese stark divergierenden Tendenzen mit der jeweiligen Stellung und Bedeutung der russischen Staatsduma und der Verchovna Rada der Ukraine im politischen System?

7 Der Begriff der „Domestizierung“ wurde von Andreas Schedler übernommen, der damit eine Strategie autoritärer Vereinnahmung formaldemokratischer Institutionen beschreibt. Vgl. ders., The New Institutionalism in the Study of Authoritarian Regimes, in: Totalitarismus und Demokratie, 6. Jg. (2009), H. 2, S. $323-340$, S. 323 f.

8 Vgl. Andreas Umland, Orange Revolution als Scheideweg: Demokratisierungsschub in der Ukraine, Restaurationsimpuls in Russland, in: Osteuropa, 59. Jg. (2009), H. 11, S. $109-120$.

9 Anders Ålund / Michael McFaul, Revolution in Orange: The Origins of Ukraine's Democratic Breakthrough, Washington 2006.

10 Diese Klassifikation entspricht dabei dem Modell nach Matthew S. Shugart / John M. Carey, Presidents and Assemblies. Constitutional Design and Electoral Dynamics, Cambridge 1992, S. $23 \mathrm{ff}$.

11 Russland wird hingegen seit 2005 als „nicht frei“ eingestuft. Siehe die jährlichen Untersuchungen „Freedom in the World“, http://www.freedomhouse.org (Abruf am 24. November 2011), in denen alle Staaten den Kategorien „frei“, „teilweise frei“ und „nicht frei“ zugeordnet werden. Die Einordnung erfolgt dabei nach der Bestimmung „politischer Rechte“ und „bürgerlicher Freiheiten" auf einer Skala von 1 (Maximum) bis 7 (Minimum). Diese beiden Kategorien werden dabei untergliedert in: A - den Wahlprozess, B - politischen Pluralismus und Partizipation, C - Regierungsfunktionen und -effizienz (A - C: ,politische Rechte“) sowie D - Glaubens- und Redefreiheit, E - Vereinigungsfreiheit und den politischen Organisationsgrad, F - Rechtsstaatlichkeit, G - freie Willensbildung und individuelle Rechte (D - F: „bürgerliche Freiheiten“). Als „frei“ werden alle Staaten eingestuft, deren Mittelwert aus beiden Komponenten 2,5 nicht überschreitet. „Teilweise freie“ Staaten liegen zwischen 3,0 bis 5,0. Ab dem Wert 5,5 gelten Staaten als "nicht frei".

12 Vgl. Alexander J. Motyl, Russia’s Systemic Transformations since Perestroika: From Totalitarianism to Authoritarianism to Democracy - to Fascism?, in: The Harriman Review, 17. Jg. (2010), H. 2, S. 1 - 14; Richard Sakwa, The Dual State in Russia, in: Post-Soviet Affairs, 26. Jg. (2010), H. 3 , S. $185-206$. 


\section{Wechselbeziehungen zwischen Regierungssystemen und Regimetypen}

Die Regierungssysteme Russlands und der Ukraine werden auf Grundlage der Verfassungsnorm überwiegend als semipräsidentiell qualifiziert. ${ }^{13}$ Einige Autoren geben jedoch zu Recht zu bedenken, dass eine derartige typologische Zuordnung aufgrund der besonderen politischen Entwicklungsdynamiken und kaum ausgeprägter konstitutioneller Traditionen in Osteuropa nicht bedenkenlos vorzunehmen ist. ${ }^{14}$ Die in den postkommunistischen Verfassungen selbst bereits angelegte Überhöhung des Präsidentenamtes ist für den postsowjetischen Raum als „Superpräsidentialismus"15 beschrieben worden. Dieser Begriff kann jedoch insofern in die Irre führen, als eine Nähe zu präsidentiellen Regierungssystemen suggeriert wird. Tatsächlich aber hat der "Superpräsidentialismus" mit präsidentiellen Regierungssystemen ebenso wenig wie mit dem Semipräsidentialismus gemein: In präsidentiellen Regierungssystemen besteht aufgrund der strikten Gewaltentrennung keine Rücktrittsverpflichtung der Regierung als Konsequenz aus einem parlamentarischen Misstrauensvotum. Genau dies ist jedoch zumindest formal in Russland und der Ukraine durch die Verfassung vorgesehen. ${ }^{16}$ Ein weiteres Indiz betrifft das Auflösungsrecht des Präsidenten gegenüber der Legislative. Während dies in präsidentiellen Regierungssystemen grundsätzlich nicht gegeben ist, muss der russische Staatspräsident nach dreimaliger Ablehnung seines Wunschkandidaten für den Posten des Premierministers das Parlament auflösen. In der Ukraine werden die Umstände und Voraussetzungen einer vorzeitigen Auflösung der Verchovna Rada durch den Präsidenten detailliert im Verfassungsartikel 90 vorgeschrieben.

Anhand der Definitionsmerkmale des Semipräsidentialismus kann ebenfalls verdeutlicht werden, dass selbst eine ausschließlich auf Grundlage der Verfassungsnorm erfolgende Zuordnung Russlands und der Ukraine zu diesem unzutreffend ist. Semipräsidentialismus wird hier verstanden als: „A regime where there is both a popularly elected fixed-term president and a prime minister and cabinet responsible to the legislature. " 17 Dabei ist für den postsowjetischen Raum eher von einer „unechten“ dualen Exekutive zu sprechen. ${ }^{18}$ Sowohl in Russland als auch der Ukraine sind die Regierungsbildung und -abberufung nur bedingt an parlamentarische Mehrheiten gekoppelt. So hat der russische Präsident ein exklusives

13 Vgl. Oleh Protsyk, Troubled Semi-Presidentialism: Stability of the Constitutional System and Cabinet in Ukraine, in: Europe-Asia Studies, 55. Jg. (2003), H. 7, S. 1077 - 1095; Sylvia von Steinsdorff, Die Verfassungsgenese der Zweiten Russischen und der Fünften Französischen Republik im Vergleich, in: ZParl, 26. Jg. (1995), H. 3, S. 486 - 504.

14 Vgl. William A. Clark, Boxing Russia: Executive-Legislative Powers and the Categorization of Russia’s Regime Type, in: Demokratizatsiya, 19. Jg. (2011), H. 1, S. 5 - 22; Kimitaka Matsuzato, Semipresidentialism in Ukraine: Institutionalist Centrism in Rampant Clan Politics, in: Demokratizatsiya, 13. Jg. (2005), H. 1, S. $45-60$.

15 John T. Ishiyama / Ryan Kennedy, Superpresidentialism and Political Party Development in Russia, Ukraine, Armenia and Kyrgyzstan, in: Europe-Asia Studies, 53. Jg. (2001), H. 8, S. 1177 - 1193.

16 Siehe Art. 103, Abs. 1; Art. 117, Abs. 3 (russische Verfassung) sowie Art. 87; Art. 115, Abs. 1 (ukrainische Verfassung vor und nach 2006).

17 Robert Elgie / Sophia Moestrup, Semi-Presidentialism: A Common Regime Type, But One That Should Be Avoided?, in: dies. (Hrsg.), Semi-Presidentialism in Central and Eastern Europe, Manchester 2008, S. 1 - 13, S. 4.

18 Vgl. Kimitaka Matsuzato, Differing Dynamics of Semipresidentialism across Euro / Eurasian Borders: Ukraine, Lithuania, Poland, Moldova, and Armenia, in: Demokratizatsiya, 14. Jg. (2006), H. 3, S. $317-346$, S. 317 f. 
Vorschlagsrecht für den Posten des Premierministers. Daher dominierten insbesondere während der Präsidentschaft Vladimir Putins unter den Premierministern und Kabinettsmitgliedern nicht dem Parlamentsumfeld angehörende, dem Präsidenten loyal ergebene "Technokraten" ${ }^{19}$ Auch in der Ukraine konnten Premierminister unter der Präsidentschaft Leonid Kucmas lediglich zum Ende dessen zweiter Amtszeit (1999 bis 2004) dank einer parlamentarischen "Hausmacht“ ein gewisses Gegengewicht zum Präsidenten entfalten. ${ }^{20}$ Die Regierungen in beiden Ländern sind somit tendenziell eher dem Präsidenten verantwortlich als dem Parlament.

In diesem Kontext sei hier explizit darauf hingewiesen, dass sich die Bestimmung parlamentarischer, präsidentieller oder semipräsidentieller Regierungssysteme lediglich auf liberale, rechts- und verfassungsstaatlich eingehegte Demokratien beziehen kann. Bei Russland und der Ukraine handelt es sich hingegen um quasikonstitutionelle Regimeformen, in denen elementare Grundlagen der Demokratie - Gewaltenteilung, Rechtsstaatlichkeit, politische Verantwortlichkeit, breite politische Teilhaberechte und Pluralismus - eingeschränkt, unterwandert oder ausgesetzt werden.

\section{Institutionelle Fassaden im "neuen“ Autoritarismus}

Die „vierte Welle“21 hat im postkommunistischen Osteuropa sehr unterschiedliche Regimetypen hervorgebracht. In den Nachfolgestaaten der Sowjetunion - mit Ausnahme des Baltikums - dominieren dabei „Systeme der gescheiterten Demokratisierung" ${ }^{22}$. Im Zuge der postkommunistischen Systemtransformation sind dabei autoritäre Regime entstanden, die die Institutionenarchitektur liberaler Demokratien „imitieren“. Obwohl formaldemokratische institutionelle Komponenten gegeben sind, vollzieht sich in „neuen“ Autoritarismen deren systematische Aushöhlung und Umdeutung. Die Wesensmerkmale des Autoritarismus sind in diesen Systemen deutlich ausgeprägt. Nach Juan J. Linz sind dies ein eingeschränkter, politisch nicht-verantwortlicher Pluralismus; ausgedehnte, jedoch nicht unbegrenzte Herrschaftsreichweite; fehlende politische Mobilisierung sowie das Vorherrschen spezifischer Mentalitäten. ${ }^{23}$ Inzwischen trägt der „neue Institutionalismus“ in der Autoritarismusforschung ${ }^{24}$ den zunehmend diffuser werdenden Stützen und Formen autoritärer Herrschaft Rechnung und untersucht, inwieweit Wahlen, Verfassungen und Verfassungsgerichte, Rechts- und Justizwesen, Parlamente und Mehrparteiensysteme, zivil-

19 Vgl. Eugene Huskey, Elite Recruitment and State-Society Relations in Technocratic Authoritarian Regimes: The Russian Case, in: Communist and Post-Communist Studies, 43. Jg. (2010), H. 4, S. $363-373$.

20 Vgl. Oleh Protsyk, Intra-Executive Competition between President and Prime Minister: Patterns of Institutional Conflict and Cooperation under Semi-Presidentialism, in: Political Studies, 54. Jg. (2006), H. 2, $219-244$.

21 Michael McFaul, The Fourth Wave of Democracy and Dictatorship: Noncooperative Transitions in the Postcommunist World, in: World Politics, 54. Jg. (2002), H. 2, S. $212-244$.

22 Jerzy Maćków, Autoritarismen oder „Demokratien mit Adjektiven“? Systeme der gescheiterten Demokratisierung, in: ZPol, 10. Jg. (2000), H. 4, S. 1471 - 1499, S. 1484 ff.

23 Vgl. Juan J. Linz, Totalitarian and Authoritarian Regimes, in: Fred I. Greenstein / Nelson Polsby (Hrsg.), Handbook of Political Science, Band 3, Reading 1975, S. 175 - 411, S. 264.

24 Vgl. Andreas Schedler, a.a.O. (Fn. 7). 
gesellschaftliche Akteure oder föderale Strukturen autoritären Amtsinhabern handlungsermöglichende Ressourcen eröffnen können. ${ }^{25}$ Diese Institutionen verfolgen dabei primär das Ziel, die Erwartungssicherheit entscheidungsoffener politischer Prozesse zugunsten der Amtsinhaber zu erhöhen. Hier kommt das den Autoritarismus kennzeichnende Kernkriterium eines eingeschränkten politischen Pluralismus zur vollen Geltung. Russland und die Ukraine werden vor diesem Hintergrund hier als ,institutionalisierte Autoritarismen “26 qualifiziert. Ein solches Vorgehen trifft in der Debatte um die Verortung von Regimetypen im postsowjetischen Raum nicht auf ungeteilte Zustimmung. Bisher sind die ambivalenten Demokratisierungsprozesse im postkommunistischen Osteuropa in der Transformationsforschung häufig mit Konzepten hybrider Systeme ${ }^{27}$ oder defekter Demokratien ${ }^{28}$ erfasst worden. Hingegen zeigen die Beispiele der Staatsduma und der Verchovna Rada, wie die Funktionslogik unabhängiger Legislativorgane durch autoritäre Regierungspraktiken vereinnahmt wird. Sie können als „domestiziert" gelten. Mit diesem Begriff ${ }^{29}$ wird die von Exekutivorganen formal wie informal betriebene, intendierte Schwächung von Parlamentsmacht bezeichnet. Parlamentsmacht geht dabei weit über die verfassungsrechtlich verankerten Zuständigkeiten und Befugnisse von Parlamenten hinaus.

Die konstitutionell verankerte Stellung der Parlamente wird in Tabelle 1 mithilfe des „Parliamentary Powers Index“ verdeutlicht. ${ }^{30}$ Danach verfügt die Verchovna Rada der Ukraine über ein höheres Maß konstitutionell garantierter Parlamentsmacht als die russische Staatsduma. Dies gilt insbesondere seit der zum 1. Januar 2006 unter Präsident Viktor Jušcenko in Kraft getretenen Verfassungsreform, die vor allem die parlamentarische Kernfunktion der Regierungsbildung und -abberufung stärkte. Diese Kräfteverschiebung wurde allerdings auf Bestreben seines Nachfolgers Viktor Janukovyč (seit Februar 2010) durch eine

25 Vgl. Jason Brownlee, Authoritarianism in an Age of Democratization, Cambridge 2007; Jennifer Gandhi, Political Institutions under Dictatorship, Cambridge 2008; Andreas Schedler, Authoritarianism's Last Line of Defense, in: Journal of Democracy, 21. Jg. (2010), H. 1, S. $69-80$.

26 Dieser Begriff orientiert sich an der Unterscheidung von postkommunistischen „Übergangsautoritarismen“ und „institutionalisierten, posttotalitären Autoritarismen“. Letztere Form ergibt sich aus einem konsenslosen Übergangsautoritarismus und kann als quasidemokratisch und nichtrechtsstaatlich gelten. Jerzy Maćków, Die posttotalitären Autoritarismen: Die Entwicklungen und Varianten, in: ders. (Hrsg.), Autoritarismus in Mittel- und Osteuropa, Wiesbaden 2009, S. 325 -347, S. $345 \mathrm{ff}$.

27 Vgl. Matthijs Bogaards, How to Classify Hybrid Regimes? Defective Democracy and Electoral Authoritarianism, in: Democratization, 16. Jg. (2009), H. 2, S. 399 - 423; Larry Diamond, Thinking about Hybrid Regimes, in: Journal of Democracy, 13. Jg. (2002), H. 2, S. 21 - 34; Steven Levitsky / Lucan A. Way, Competitive Authoritarianism: Hybrid Regimes after the Cold War, Cambridge 2010.

28 Vgl. Aurel Croissant, Analyse defekter Demokratien, in: Klemens H. Schrenk / Markus Soldner (Hrsg.), Analyse demokratischer Regierungssysteme, Wiesbaden 2010, S. 93 - 114; Wolfgang Merkel, Embedded and Defective Democracies, in: Democratization, 11. Jg. (2004), H. 5, S. 33 -58 .

29 Vgl. Fußnote 7.

30 Dieser wurde von M. Steven Fish und Matthew Kroenig entwickelt (The Handbook of National Legislatures: A Global Survey, Cambridge 2009, S. 1 - 17). Die Autoren ordnen die einzelnen Indikatoren von „Parlamentsmacht“ dabei vier Kategorien zu: (1) der Einfluss des Parlaments gegenüber der Exekutive; (2) die institutionelle Autonomie des Parlaments; (3) konstitutionell garantierte Zuständigkeiten und Befugnisse des Parlaments; (4) die institutionelle Leistungsstärke und Ausstattung. 


\begin{tabular}{|c|c|c|c|}
\hline \multirow[b]{2}{*}{$\begin{array}{l}\text { Konstitutionelle Vollmachten der Legislative und deren Stel- } \\
\text { lung im politischen System }\end{array}$} & \multirow[b]{2}{*}{ Russland } & \multicolumn{2}{|c|}{ Ukraine } \\
\hline & & $\begin{array}{l}1996 \\
\text { bis } 2005 \\
\text { seit } 2010\end{array}$ & $\begin{array}{c}2006 \\
\text { bis } 2010\end{array}$ \\
\hline Einfluss und Kontrollmechanismen über Exekutive & $1 / 9$ & $2 / 9$ & $4 / 9$ \\
\hline $\begin{array}{l}\text { 1. Amtsenthebung des Präsidenten ohne Einbeziehung einer } \\
\text { anderen Institution oder Absetzung des Premierministers }\end{array}$ & & & \\
\hline $\begin{array}{l}\text { 2. Mitglieder des Ministerkabinetts sind Abgeordnete des } \\
\text { Parlaments }\end{array}$ & & & \\
\hline $\begin{array}{l}\text { 3. Vorladungs- und Anhörungsrechte gegenüber Vertretern } \\
\text { von Exekutivorganen }\end{array}$ & & $\mathrm{x}$ & $\mathrm{x}$ \\
\hline $\begin{array}{l}\text { 4. Recht auf unabhängige Ermittlungen gegenüber Exekuti- } \\
\text { vorganen }\end{array}$ & & & \\
\hline $\begin{array}{l}\text { 5. Aufsicht über Sicherheitsstrukturen (Militär, Vollzugsbe- } \\
\text { hörden, Geheimdienst, Polizei) }\end{array}$ & & & \\
\hline 6. Ernennung des Regierungschefs & & & $\mathrm{x}$ \\
\hline $\begin{array}{l}\text { 7. Bestimmung oder Zustimmung zur Ernennung einzelner } \\
\text { Minister }\end{array}$ & & & $\mathrm{x}$ \\
\hline 8. Kein Präsidentenamt oder dessen Wahl durch Parlament & & & \\
\hline $\begin{array}{l}\text { 9. Parlamentarisches Misstrauensvotum gegenüber Regie- } \\
\text { rung ohne gleichzeitige Auflösung des Parlaments }\end{array}$ & $\mathrm{x}$ & $\mathrm{x}$ & $\mathrm{x}$ \\
\hline Institutionelle Autonomie & $3 / 9$ & $4 / 9$ & $4 / 9$ \\
\hline $\begin{array}{l}\text { 10. Kein Auflösungsrecht der Exekutive gegenüber dem Par- } \\
\text { lament }\end{array}$ & & & \\
\hline $\begin{array}{l}\text { 11. Keine Legislativrechte der Exekutive ohne Zustimmung } \\
\text { des Parlaments }\end{array}$ & & & \\
\hline $\begin{array}{l}\text { 12. Kein Vetorecht der Exekutive oder Veto mit einfacher } \\
\text { oder Mehrheit im Parlament zu überstimmen }\end{array}$ & & & \\
\hline 13. Gesetzgebung unterliegt nicht richterlicher Überprüfung & & & \\
\hline 14. Gesetzgeberische Initiativrechte in allen Politikfeldern & $\mathrm{x}$ & $\mathrm{x}$ & $\mathrm{x}$ \\
\hline $\begin{array}{l}\text { 15. Aufwendungen für Parlamentsbetrieb zwingend vorgese- } \\
\text { hen und nicht durch Exekutive korrigierbar }\end{array}$ & & & \\
\hline $\begin{array}{l}\text { 16. Parlament hat selbst Aufsicht über die Verwendung von } \\
\text { Mitteln für den Arbeitsprozess und die Ausstattung der } \\
\text { Abgeordneten }\end{array}$ & & $\mathrm{x}$ & $\mathrm{x}$ \\
\hline 17. Abgeordnetenimmunität & $\mathrm{x}$ & $\mathrm{x}$ & $\mathrm{x}$ \\
\hline 18. Alle Abgeordneten sind gewählt & $\mathrm{x}$ & $\mathrm{x}$ & $\mathrm{x}$ \\
\hline Vollmachten und Befugnisse & $4 / 8$ & $6 / 8$ & $6 / 8$ \\
\hline $\begin{array}{l}\text { 19. Parlament kann ohne Mitwirkung anderer Institutionen } \\
\text { Verfassung ändern }\end{array}$ & & & \\
\hline 20. Kriegserklärung von Parlament zu bestätigen & $\mathrm{x}$ & $\mathrm{x}$ & $\mathrm{x}$ \\
\hline 21. Parlament muss internationalen Verträgen zustimmen & $\mathrm{x}$ & $\mathrm{x}$ & $\mathrm{x}$ \\
\hline 22. Kann Amnestie gewähren & $\mathrm{x}$ & $\mathrm{x}$ & $\mathrm{x}$ \\
\hline 23. Kann Begnadigungen aussprechen & & & \\
\hline
\end{tabular}




\begin{tabular}{|c|c|c|c|}
\hline \multicolumn{4}{|l|}{ Fortsetzung Tabelle 1} \\
\hline $\begin{array}{l}\text { 24. Ernennung von Richtern oder Zustimmung zu deren Er- } \\
\text { nennung }\end{array}$ & $\mathrm{x}$ & $\mathrm{x}$ & $\mathrm{x}$ \\
\hline 25. Ernennt Vorsitzenden der Zentralbank & & $\mathrm{x}$ & $\mathrm{x}$ \\
\hline 26. Mitsprache über Arbeitsweise staatlicher Medien & & $\mathrm{x}$ & $\mathrm{x}$ \\
\hline Institutionelle Leistungsfähigkeit und Ausstattung & $6 / 6$ & $5 / 6$ & $5 / 6$ \\
\hline 27. Reguläre Sitzungsperioden & $\mathrm{x}$ & $\mathrm{x}$ & $\mathrm{x}$ \\
\hline 28. Jeder Abgeordnete hat Recht auf Büroangestellten & $\mathrm{x}$ & $\mathrm{x}$ & $\mathrm{x}$ \\
\hline 29. Jeder Abgeordnete hat zumindest einen Assistenten & $\mathrm{x}$ & & \\
\hline $\begin{array}{l}\text { 30. Abgeordnete können sich ohne Einschränkungen zur } \\
\text { Wiederwahl stellen }\end{array}$ & $\mathrm{x}$ & $\mathrm{x}$ & $\mathrm{x}$ \\
\hline 31. Attraktivität des Abgeordnetenmandats & $\mathrm{x}$ & $\mathrm{x}$ & $\mathrm{x}$ \\
\hline 32. Qualifizierter und erfahrener Abgeordnetenkorpus & $\mathrm{x}$ & $\mathrm{x}$ & $\mathrm{x}$ \\
\hline Gesamt & .44 & .53 & .59 \\
\hline \multicolumn{4}{|c|}{$\begin{array}{l}\text { Quelle: Eigene Darstellung nach M. Steven Fish / Matthew Kroenig, a.a.O. (Fn. 30). Martin Sebald } \\
\text { schlägt eine ähnliche Vorgehensweise anhand der Messung von Parlamentsfunktionen vor und unter } \\
\text { scheidet: Kreationsfunktion, Gesetzgebungsfunktion, Kontrollfunktion, Repräsentations- und Kommu- } \\
\text { nikationsfunktion. Vgl. Martin Sebaldt, Die Macht der Parlamente. Funktionen und Leistungsprofil } \\
\text { nationaler Volksvertretungen in den alten Demokratien der Welt, Wiesbaden 2009, S. } 21 \text { - } 27 \text {. }\end{array}$} \\
\hline
\end{tabular}

umstrittene Entscheidung des ukrainischen Verfassungsgerichts vom 30. September 2010 wieder aufgehoben. Seither herrscht Unklarheit darüber, ob damit die ursprüngliche Verfassung von 1996 erneut ihre Rechtsgültigkeit in vollem Umfang wiedererlangt hat. ${ }^{31}$

Es ist zu berücksichtigen, dass beide hier behandelten Fälle keine liberalen, konstitutionell eingehegten Rechts- und Verfassungsstaaten sind. Das Bestehen einer Verfassung allein kann nicht die Befolgung konstitutioneller Prinzipien im Sinne von „limited government“ garantieren. ${ }^{32}$ Der in Tabelle 1 dargestellte „Parliamentary Powers Index“ stellt lediglich die in der Verfassung unmittelbar enthaltenen Zuständigkeiten und Vollmachten der Staatsduma und der Verchovna Rada dar. Problematisch ist dabei, dass die insgesamt 32 aufgeführten Punkte nicht gemäß ihrer unterschiedlichen Relevanz und Ausprägung gewichtet werden, woraus sich Verzerrungen und Fehldeutungen ergeben können.

Der Machtbegriff muss somit ausdifferenziert werden: Zunächst stehen sich hier zwei Dimensionen gegenüber, die exemplarisch auf Max Weber (transitives Machtverständnis im Sinne von "power over") und Hannah Arendt (intransitives Machtverständnis im Sinne von "power to") zurückgehen. ${ }^{33}$ Beide Aspekte verbinden sich in der von Werner Patzelt vorgeschlagenen Differenzierung von „Durchsetzungsmacht“, „Vetomacht“ und „Deutungsmacht“. Eine derartige Untergliederung trägt dem Umstand Rechnung, dass politische Macht häufig diffuse und „unsichtbare“ Formen annimmt. Zudem ergibt sich aus der Ge-

31 Vgl. European Commission for Democracy through Law, Opinion on the Constitutional Situation in Ukraine, Opinion No. 599/2010 vom 17./18. Dezember 2010, http://www.venice.coe. int/docs/2010/CDL-AD\%282010\%29044-e.asp (Abruf am 24. November 2011).

32 Vgl. Jon Elster, Constitutionalism in Eastern Europe: An Introduction, in: University of Chicago Law Review, 58. Jg. (1991), H. 2, S. 447 - 482, S. 465 ff.

33 Vgl. Gerhard Göhler, Macht, in: ders. / Mattias Iser / Ina Kerner (Hrsg.), Politische Theorie. 22 umkämpfte Begriffe, Wiesbaden 2004, S. $244-261$. 
währung konstitutionell verankerter Kompetenzen und Befugnisse („capacity“) nicht zwangsläufig auch deren Ausübung sowie reale Handlungsmacht („empowerment“). In diesem Kontext ist demnach auch die Unterscheidung von „Bruttomacht“, die sich aus den verfügbaren und rechtlich garantierten Handlungsressourcen politischer Akteure und Institutionen ergibt, und der "Nettomacht" relevant, die die "Gegenmacht" in Form von Transaktionskosten sowie unvorhersehbaren Konsequenzen bei der Ausübung politischer Macht berücksichtigt und antizipiert. ${ }^{34}$ Durchsetzungsmacht und Vetomacht eines Parlaments offenbaren sich insbesondere bei Konfliktsituationen im Verhältnis zu Exekutivorganen. Erstere kommt beispielsweise bei der Regierungsbildung und -abberufung sowie bei der Gesetzgebungsfunktion zur Geltung: Ist das Parlament fähig, seine Positionen ungeachtet des Widerstands und formaler (Vetorecht des Präsidenten, Dekretrechte, Anberaumung von Referenda, Parlamentsauflösung) wie informaler Beeinflussung durchzusetzen? Vetomacht wird im Falle der Missbilligung des Regierungsprogramms, der Verweigerung der Zustimmung bei der Ernennung des Generalstaatsanwalts, des Vorsitzes der Nationalbank oder weiterer staatlicher Behörden und Gremien ausgeübt. In der parlamentarischen Deutungsmacht kommt in erster Linie eine bestimmte institutionelle Autorität und Dignität zum Ausdruck, die eine vor allem ethisch-moralische handlungsbindende Wirkung bei der politischen Entscheidungsbildung entfalten kann. Parlamentsmacht wird somit als Summe der formalen wie informalen, empirisch nachweisbaren wie „unsichtbaren“, sowohl transitiv als auch intransitiv wirkenden politischen Handlungsmacht einer Vertretungskörperschaft verstanden, die sich zudem analytisch in Durchsetzungsmacht, Vetomacht und Deutungsmacht unterteilen lässt.

\section{Strategien der "Domestizierung" von Parlamenten im Postkommunismus}

Das Beziehungsgeflecht zwischen Präsidenten und Legislativorganen ist eine der zentralen Determinanten bei der Herausbildung postkommunistischer Regierungssysteme und Regimetypen. Im postsowjetischen Raum standen sich unmittelbar nach der Auflösung der Sowjetunion 1991 vermeintlich „reformunwillige“, durch starke kommunistische Fraktionen oder deren Nachfolgeparteien dominierte Parlamente und „reformfreudige“ Präsidenten gegenüber. Die ersten freien und weitestgehend fairen Parlamentswahlen fanden dabei in Russland 1993 und in der Ukraine im Jahr darauf statt. Aus dieser Rollenverteilung heraus sahen sich die Präsidenten in beiden Ländern bald veranlasst, eine institutionelle Einhegung der Legislative zu betreiben. Bei dieser „Domestizierung“ wurden verschiedene Strategien verfolgt: (1) die Entmachtung der Parlamente („disempowerment“), (2) die Kontrolle und Beeinflussung der Abgeordneten (,agent control“) und (3) die Fragmentierung und Desorganisation (,fragmentation“). ${ }^{35}$ Ziel dabei ist, die Durchsetzungsmacht, Vetomacht und Deutungsmacht der Parlamente zu beschränken. 


\subsection{Entmachtung der Parlamente („disempowerment“)}

Sowohl in Russland als auch in der Ukraine haben sich die Präsidenten in der Auseinandersetzung mit „widerspenstigen“ Legislativorganen verfassungspolitischer Mittel bedient. Ausgangspunkt der jüngeren russischen Verfassungsgeschichte bildet ein Konflikt zwischen der Staatsduma und dem damaligen Präsidenten Boris El'cin (1991 bis 1999), den dieser 1993 durch die Auflösung des Parlaments, die Erstürmung des Parlamentsgebäudes sowie ein anschließendes Referendum über einen von ihm ausgearbeiteten Verfassungsentwurf für sich entscheiden konnte. ${ }^{36}$ Die weitere verfassungspolitische Entwicklung des Landes zeichnet sich durch die nahezu vollständige politische Vereinnahmung des Rechts- und Justizwesens unter den Präsidenten Vladimir Putin und Dmitrij Medvedev aus. Bei politisch kontroversen Entscheidungen - wie dem Yukos-Prozess, der seit 2005 praktizierten Ernennung der Gouverneure der Föderationssubjekte durch den Präsidenten und der Abschaffung der Wahl von Bürgermeistern, der folgenschweren Änderung der Wahlgesetzgebung sowie der Verlängerung der Amtszeit des Präsidenten - wurde formal die Verfassungsmäßigkeit bei diesen Maßnahmen gewahrt beziehungsweise gegebenenfalls die Verfassungskonformität hergestellt. ${ }^{37}$ Die Exekutive besitzt weitestgehend die Deutungshoheit über die Verfassung, so dass insbesondere das russische Verfassungsgericht zunehmend eher als willenloser „Sklave der Verfassung “ denn als autonomer Akteur agiert. ${ }^{38}$

Das Ensemble konzertierter Institutionen wird durch die zwei Kammern des Parlaments komplettiert. Während die Vertreter des Föderationsrates seit 2004 vom Präsidenten handverlesen werden, wird die Staatsduma trotz der Stimmeneinbußen bei der jüngsten Parlamentswahl vom 4. Dezember 2011 von der Partei „Einiges Russland“ („Edinaja Rossija“) dominiert. Seit der Parlamentswahl 2007 stellte diese 315 der 450 Abgeordneten. In allen Regionalparlamenten der 83 Föderationssubjekte verfügt die Partei wenigstens über eine relative Mehrheit. Vladimir Putin übt seit Übernahme des Premierministerpostens im April 2008 den Parteivorsitz aus, ohne jedoch selbst Mitglied zu sein. Der Parlamentspräsident Boris Gryslov, der bereits 2007 befand, die Staatsduma sei „nicht der richtige Platz für Diskussionen “39, bezog auch in der Frage der Bestimmung der Kandidatur für die Präsidentschaftswahl 2012 eine Position, die symptomatisch für die Ergebenheit der Staatsduma gegenüber der Exekutive ist. Demnach würde das aus Premier Putin und Präsident Medvedev bestehende „Tandem“ auch künftig die Staatsgeschäfte führen. ${ }^{40}$

Die Funktionen, Zuständigkeiten und Instrumentarien von Parlamentariern der Staatsduma unterscheiden sich formal nur unwesentlich von denen ihrer westeuropäischen Kollegen. Ihre Kontroll- und Vetomöglichkeiten gegenüber der Exekutive wenden die Abgeordneten jedoch nicht vorrangig im Sinne einer normativen, funktionalen Regierungs-

36 Vgl. Steven Levitsky / Lucan A. Way, a.a.O. (Fn. 27), S. 190 ff.

37 Vgl. Margareta Mommsen, Das politische System Russlands, in: Wolfgang Ismayr (Hrsg.), Die politischen Systeme Osteuropas, Wiesbaden 2010, S. 419 - 478, S. $461 \mathrm{ff}$.

38 Vgl. Angelika Nußberger, Das Russische Verfassungsgericht zwischen Recht und Politik, in: Matthes Bubbe / Gabriele Gorzka (Hrsg.), Russland heute. Rezentralisierung des Staates unter Putin, Wiesbaden 2007, S. 215 - 233, S. 215 f.

39 Aleksej Levčenko, Nemesto dlja diskussij, 15. November 2007, http://www.gazeta.ru/politics/elections2007/articles/2311346.shtml (Abruf am 24. November 2011).

40 Marija Cybul'skaja, Tandem budet i Poste 2012 goda, 19. März 2010, http:77www.gazeta.ru/interview/nm/s3337459.shtml (Abruf am 24. November 2011). 
kontrolle an, sondern vielmehr nach einem Kalkül der Verwertbarkeit und Konvertierbarkeit dieser potenziellen Vetomacht zur Verfolgung individueller Interessen einzelner oder einer Gruppe von Parlamentariern. Die neopatrimoniale Logik dieser Mechanismen ist dadurch gekennzeichnet, dass die Zunahme und Heterogenisierung institutionalisierter parlamentarischer Handlungsressourcen - so bei der Anzahl parlamentarischer Komitees, der Einflussnahme auf das Agenda Setting oder der Bildung von Gruppen innerhalb von Parlamentsfraktionen - mit der gleichzeitigen funktionalen Aushöhlung der Staatsduma als Institution vereinbar sind. ${ }^{41}$ Die Ende 2008 implementierten ersten substanziellen, verfassungsrechtlich problematischen Zusätze zur Verfassung von 1993 sind Ausdruck der Gefügigkeit der Staatsduma gegenüber der Exekutive: Innerhalb weniger Wochen wurde die Amtszeit des Präsidenten von vier auf sechs Jahre und die Wahlperiode der Staatsduma von vier auf fünf Jahre ausgedehnt. ${ }^{42}$

Im Gegensatz zu dieser Entwicklung blieben die Bemühungen des ukrainischen Präsidenten Leonid Kučma um eine konstitutionelle Einhegung der Legislative weitestgehend erfolglos. Hierin bestand ein ganz wesentlicher Bedingungsfaktor der Orangenen Revolution 2004. Im April 2000 wurden sechs das künftige Institutionengefüge des Regierungssystems betreffende Fragen auf Initiative des Präsidenten dem Volk zur Abstimmung gestellt. ${ }^{43}$ Die Bestimmungen dieses Verfassungsreferendums wurden allerdings von den Abgeordneten der Verchovna Rada nie ratifiziert. Die wachsende Unpopularität des Präsidenten, eine gut organisierte parlamentarische Opposition sowie nicht zuletzt die außenpolitische Isolation der Ukraine in der Spätphase Kučmas zweiter Amtszeit (1999 bis 2004) ließen eine ähnliche Entwicklung wie in Russland nicht zu. ${ }^{44}$ Nach der Amtsübernahme durch Viktor Jušcenko trat 2006 eine im Zuge der Orangenen Revolution ausgehandelte Verfassungsre-

41 Vgl. Sarah Whitmore, Parliamentary Oversight in Putin's Neo-patrimonial State. Watchdogs or Show-dogs?, in: Europe-Asia Studies, 62. Jg. (2010), H. 6, S. 999 - 1025, S. 1002 ff.; Thomas Remington, Patronage and the Party of Power: President-Parliament Relations under Vladimir Putin, in: Europe-Asia Studies, 60. Jg. (2008), H. 6, S. 959 - 987, S. 960 ff.

42 Vgl. Mark Mandelbaum, Die Änderung der russischen Verfassung - harmlose Korrektur oder „verfassungswidriges Verfassungsrecht"?, in: Jahrbuch für Ostrecht, 50. Jg. (2009), H. 2, S. 311 $-328$.

43 Im Einzelnen standen folgende Verfassungsänderungen bei dem Referendum zur Debatte: (1) die Möglichkeit der Auflösung der Verchovna Rada durch ein öffentliches Misstrauensvotum der Bevölkerung; (2) die Ratifizierung von Verfassungsänderungen durch Referenda; (3) die Auflösung der Verchovna Rada durch den Präsidenten für den Fall, dass über einen Zeitraum von 30 Tagen keine parlamentarische Mehrheit besteht oder der Staatshaushalt drei Monate nach Einbringen durch die Regierung nicht durch das Parlament bestätigt wird; (4) die Aufhebung der Abgeordnetenimmunität und weiterer Privilegien von Parlamentariern; (5) die Reduzierung der Abgeordnetenmandate der Verchovna Rada von 450 auf 300; (6) die Etablierung einer zweiten Parlamentskammer. Alle Vorschläge des Präsidenten wurden von einer überwältigenden Mehrheit gebilligt. Die ersten beiden Formulierungen wurden allerdings umgehend vom Verfassungsgericht annulliert. Vgl. Oleh Protsyk, Constitutional Politics and Presidential Power in Kuchma's Ukraine, in: Problems of Post-Communism, 52. Jg. (2005), H. 5, S. 23 - 31.

44 Leonid Kučma wurde beschuldigt, die Ermordung des Journalisten Gongadze angewiesen zu haben. Die folgenden Massenproteste 2001 („Ukraine ohne Kučma!“) können dabei in gewisser Hinsicht bereits als Vorboten der Orangenen Revolution 2004 gelten. Darüber hinaus ergab sich ein schwerer internationaler Imageverlust der ukrainischen Staatsführung aus dem illegalen Export von Radarsystemen des Typs „Kolčuga“ in den Irak. Vgl. Ingmar Bredies, Institutionenwandel ohne Elitenwechsel? Das ukrainische Parlament im Kontext des politischen Systemwechsels 1990-2006, Hamburg 2007, S. 183 - 195. 


\begin{tabular}{|c|c|c|c|}
\hline \multirow{2}{*}{$\begin{array}{l}\text { Konstitutionelle Vollmachten des } \\
\text { Präsidenten }\end{array}$} & \multirow[b]{2}{*}{ Russland } & \multicolumn{2}{|c|}{ Ukraine } \\
\hline & & $\begin{array}{l}1996 \text { bis } 2005 \\
\text { seit } 2010\end{array}$ & 2006 bis 2010 \\
\hline \multicolumn{4}{|l|}{ Legislative Befugnisse* } \\
\hline Vetorecht (package veto) & 2 & 2 & 2 \\
\hline $\begin{array}{l}\text { Vetorecht gegen Einzelbestimmungen } \\
\text { (line-item veto) }\end{array}$ & 0 & 0 & 0 \\
\hline Dekretrechte & 4 & 4 & 1 \\
\hline Exklusive legislative Initiativrechte & 0 & 0 & 0 \\
\hline Staatshaushalt & 0 & 0 & 0 \\
\hline Ansetzung von Referenda & 0 & 2 & 2 \\
\hline \multicolumn{4}{|l|}{ Nicht-legislative Befugnisse** } \\
\hline Regierungsbildung & 1 & 1 & 0 \\
\hline Abberufung der Regierung & 4 & 4 & 0 \\
\hline Misstrauensvotum & 2 & 0 & 0 \\
\hline Auflösung des Parlaments & 1 & 0 & 1 \\
\hline Gesamt & 14 & 13 & 6 \\
\hline \multicolumn{4}{|c|}{$\begin{array}{l}\text { * Shugart und Carey gewichten präsidentielle Vollmachten von } 0 \text { (Merkmal nicht ausgeprägt) bis } 4 \\
\text { (starke Ausprägung). Unter Berücksichtigung der hier aufgeführten legislativen Befugnisse sind diese } \\
\text { Wertigkeiten wie folgt belegt: Vetorecht ( } 4 \text { - Veto ohne Möglichkeit der Überstimmung; } 3 \text { - Veto mit } \\
\text { größerer als Zweidrittelmehrheit aufhebbar; } 2 \text { - durch Zweidrittelmehrheit überstimmbar; } 1 \text { - absolu- } \\
\text { te Mehrheit oder weniger als Zweidrittelmehrheit; } 0 \text { - kein Vetorecht oder mit einfacher Mehrheit } \\
\text { aussetzbar); Vetorecht gegen Einzelbestimmungen ( } 4 \text { - Veto ohne Möglichkeit der Überstimmung; } \\
3 \text { - qualifizierte Mehrheit; } 2 \text { - absolute Mehrheit; } 1 \text { - einfache Mehrheit; } 0 \text { - kein Vetorecht); Dekret- } \\
\text { rechte ( } 4 \text { - nicht anfechtbare Dekretrechte; } 2 \text { - vorübergehende Dekretrechte mit einigen Einschrän- } \\
\text { kungen; } 1 \text { - eingeschränkte Dekretrechte; } 0 \text { - keine eigenständigen Dekretrechte); Exklusive legislati- } \\
\text { ve Initiativrechte ( } 4 \text { - keine Möglichkeit der Abänderung; } 2 \text { - eingeschränkte Abänderung durch } \\
\text { Parlament; } 1 \text { - uneingeschränkte Abänderung durch Parlament; } 0 \text { - keine Initiativrechte); Staatshaus- } \\
\text { halt ( } 4 \text { - Vorschlag des Präsidenten ohne Änderungsmöglichkeiten; } 3 \text { - Parlament kann Haushalt } \\
\text { kürzen, jedoch nicht erhöhen; } 2 \text { - Präsident setzt Limit fest, in dessen Rahmen Parlament Nachbesse- } \\
\text { rungen vornimmt; } 1 \text { - Parlament kann Haushalt erhöhen, falls neue Staatseinnahmen zustande kom- } \\
\text { men; } 0 \text { - uneingeschränkt Nachbesserungen durch Parlament möglich); Referenda (4 - uneinge- } \\
\text { schränkt durch Präsidenten ansetzbar; } 2 \text { - mit Einschränkungen durchführbar; } 0 \text { - keine } \\
\text { Vollmachten). }\end{array}$} \\
\hline \multicolumn{4}{|c|}{$\begin{array}{l}\text { ** Für nicht-legislative Befugnisse und Vollmachten des Präsidenten ergibt sich folgende Gewichtung: } \\
\text { Regierungsbildung (4 - Präsident ernennt gesamte Regierung ohne Bestätigung oder Amtseinsetzung } \\
\text { durch Parlaments; } 3 \text { - Präsident ernennt Regierungsmitglieder bei Bestätigung oder Amtseinsetzung } \\
\text { durch das Parlament; } 1 \text { - Präsident ernennt unter Zustimmung des Parlaments Regierungschef, der } \\
\text { das Kabinett selbst bestimmt; } 0 \text { - Präsident hat keine Ernennungsrechte); Regierungsabberufung (4 } \\
\text { - Präsident kann jederzeit Minister entlassen; } 2 \text { - Entlassung von Regierungsmitgliedern mit Ein- } \\
\text { schränkungen; } 1 \text { - Entlassung nur bei Zustimmung des Parlaments für Nachfolgekandidaten/ neues } \\
\text { Kabinett; 0 - Misstrauensvotum des Parlaments gegenüber der Regierung); Misstrauensvotum (4 - } \\
\text { kein Misstrauensvotum des Parlaments gegenüber Regierung; } 2 \text { - Misstrauensvotum bei gleichzeiti- } \\
\text { gem Aufösungsrecht des Präsidenten gegenüber dem Parlament; } 1 \text { - konstruktives Misstrauensvotum; } \\
\text { 0 - uneingeschränktes Misstrauensvotum); Auflösung des Parlaments durch Präsidenten (4 - uneinge- } \\
\text { schränkte Auflösung; } 3 \text { - Einschränkungen bei Häufigkeit und Zeitpunkt; } 2 \text { - neue Präsidentschafts- } \\
\text { wahlen erforderlich; } 1 \text { - nur bei Misstrauensvotum des Parlaments gegenüber Regierung; } 0 \text { - keine } \\
\text { Vollmachten des Präsidenten). } \\
\text { Quelle: Eigene Darstellung nach Matthew S. Shugart / John M. Carey a.a.O. (Fn. 10), S. } 150 \text {. }\end{array}$} \\
\hline
\end{tabular}


form in Kraft, die eine Verlagerung politischer Kompetenzen und Zuständigkeiten zugunsten des Parlaments und der Regierung vorsah (siehe Punkte 6 und 7 in Tabelle 1). ${ }^{45}$ In dieser Periode konnten die in Tabelle 1 ausgewiesenen legislativen Kontrollmechanismen gegenüber der Exekutive erstmals reale Geltungskraft erlangen. Noch deutlicher werden die Auswirkungen der von 2006 bis 2010 gültigen Verfassungsänderungen in der Ukraine durch den „Presidential Power Index“ in Tabelle 2. Mit der umstrittenen Aufhebung der Verfassungsreform in der Ukraine unter dem amtierenden Präsidenten Viktor Janukovyč sind die Vollmachten des Staatsoberhauptes wieder restauriert worden und demnach nur unwesentlich geringer als die seines russischen Amtskollegen.

Insbesondere die Anwendung des suspensiven Vetorechts, die umfassenden Dekretrechte sowie die in der Ukraine noch immer gegebene Möglichkeit der Ansetzung von Referenda untergraben permanent die Durchsetzungs- und Vetomacht der Parlamente. ${ }^{46}$ Darüber hinaus haben informale und parakonstitutionelle Regierungspraktiken der Präsidenten in Russland und der Ukraine zur Marginalisierung der Legislative beigetragen. ${ }^{47}$

\subsection{Kontrolle und Beeinflussung der Abgeordneten (,agent control“)}

Parlamente sind kollektive Vertretungskörperschaften, deren institutionelle Autonomie, Arbeitseffizienz und Funktionalität maßgeblich von den individuellen Kompetenzen und Motivationslagen der Abgeordneten abhängen. Die Mechanismen der Rekrutierung parlamentarischer Eliten haben somit unmittelbare Folgen für das Fremd- und Selbstverständnis von Legislativorganen. Genau hier bestehen weitere Ansatzpunkte für ihre gezielte institutionelle „Domestizierung " in beiden Staaten. Sowohl in Russland als auch in der Ukraine ist über gesetzliche Rahmenbedingungen des Wahl- und Parteiensystems die Zusammensetzung der Parlamente maßgeblich verändert worden.

Ausgehend vom sowjetischen Modell der Einerwahlkreise entschieden sich beide Staaten zunächst für ein Grabensystem, das jeweils die Hälfte der 450 Sitze nach dem Mehrheitswahlrecht und dem Verhältniswahlrecht bestimmte. Die Parlamentswahlen in der Ukraine 2006 und in Russland im folgenden Jahr fanden erstmals ausschließlich nach dem Verhältniswahlrecht über geschlossene Parteilisten statt. Was auf den ersten Blick wie eine Stärkung der Rolle von Parteien und Parlament erschien, entpuppte sich in beiden Fällen als das genaue Gegenteil: Vorrangiges Ziel der „electoral (counter-)reform of 2005-2007“48 in Russland war, jeglicher Art von politischer Unvorhersehbarkeit entgegenzuwirken und die Loyalität der Abgeordneten zur Partei der Macht - „Einiges Russland“ - zu erhöhen. Restriktionen bei der Registrierung politischer Parteien - so wurden die Mindestvoraussetzungen von ursprünglich 10.000 Parteimitgliedern auf 50.000 erhöht sowie Regional-

45 Vgl. ders., Verfassungspolitik und Verfassungsentwicklung in der Ukraine unter der Präsidentschaft Viktor Juščenkos, in: Juliane Besters-Dilger / Alois Woldan (Hrsg.), Die Ukraine auf dem Weg nach Europa: Die Ära Juschtschenko, Frankfurt am Main 2011, S. 35 - 57.

46 Vgl. Oleh Protsyk, Ruling with Decrees: Presidential Decree Making in Russia and Ukraine, in: Europe-Asia Studies, 56. Jg. (2004), H. 5, S. 637 - 660.

47 Vgl. Richard Sakwa, a.a.O. (Fn. 12), S. 192 ff.; Kimitaka Matsuzato, a.a.O. (Fn. 14).

48 Stephen White / Ol'ga Kryshtanovskaya, Changing the Russian Electoral System: Inside the Black Box, in: Europe-Asia Studies, 63. Jg. (2011), H. 4, S. 557 - 578, S. 563. 
vertretungen in zwei Dritteln aller Föderationssubjekte (zuvor die Hälfte) vorausgesetzt führten in Russland zur Halbierung ihrer Gesamtzahl. Neben der bereits 2002 beschlossenen Anhebung der Eingangsbarriere von fünf auf sieben Prozent sind Wahlblöcke, in denen sich bisher mehrere Parteien zusammenschließen konnten, seither nicht mehr zu Parlamentswahlen zugelassen. Die Mindestwahlbeteiligung, die für die Gültigkeit einer Wahl bisher zwingend erforderlich war, wurde aufgehoben. Letztendlich wurde auch die viele postsowjetische Staaten kennzeichnende eigentümliche Regelung, „gegen alle“ an einer Wahl teilnehmenden politischen Kräfte stimmen zu können, abgeschafft. Hiermit wurde formal sogar einer offiziellen Empfehlung der OSZE entsprochen. ${ }^{49}$

Mit den Parlamentswahlen 2007 ist zudem der Einfluss fraktionsunabhängiger Abgeordneter mit einer autonomen, regionalen „Hausmacht“ stark geschwächt worden. Dies ist vor allem auch im Kontext der zweifelhaften „Föderalismusreformen“ zu sehen. All diese Neuerungen veränderten nachhaltig die Zusammensetzung der Staatsduma. Die Zugangskanäle ins Parlament sind aufgrund der geringen gesellschaftlichen Verankerung politischer Parteien sowie deren Monopol bei der Aufstellung der Kandidatenlisten weiter verengt worden. In Russland konnte so um „Einiges Russland“ eine effektive Machtvertikale errichtet werden, die auch die regionale und lokale Ebene durchdringt. ${ }^{50}$ Jedoch muss im Gefolge der jüngsten Parlamentswahlen wohl mit weiteren institutionellen Neujustierungen gerechnet werden.

\begin{tabular}{|l|rr|rr|rr|rr|}
\hline Tabelle 3: Ergebnisse der russischen Parlamentswahlen, 2000 bis 2011 (in Prozent) \\
\hline Partei & 2000 bis 2003 & \multicolumn{2}{|c|}{2003 bis 2007} & \multicolumn{2}{c|}{2007 bis 2011} & 2011 bis 2016 \\
& Liste & Sitze & Liste & Sitze & Liste & Sitze & Liste & Sitze \\
\hline Einheit / Einiges & 23,32 & 18,4 & 37,40 & 68,0 & 64,30 & 70,0 & 49,54 & 52,9 \\
Russland & 13,33 & 10,2 & & & & & & \\
OVR & 24,29 & 20,2 & 12,65 & 11,6 & 11,57 & 13,0 & 19,16 & 20,4 \\
KPRF & 5,98 & 3,9 & 11,49 & 8,0 & 8,14 & 9,0 & 11,66 & 12,4 \\
LDPR & 8,52 & 7,3 & 3,97 & 0 & 0,96 & 0 & & \\
SPS & 5,93 & 4,8 & 4,32 & 0 & 1,59 & 0 & 3,30 & 0 \\
Yabloko & 9,04 & 8,7 & 7,74 & 8,0 & & & 13,22 & 14,2 \\
Heimat / Gerechtes & Russland \\
Rechte Sache & Quelle: Zentrale Wahlkommission der Russischen Föderation, http://www.cikrf.ru/ (Abruf am 6. De- \\
zember 2011).
\end{tabular}

Die Errichtung einer „Partei der Macht“ - also einer weitestgehend entideologisierten parlamentarischen „Hausmacht" der Exekutive, die zudem sämtliche staatliche Ressourcen vereinnahmt und die administrativen Teilbereiche aller territorialen Gebietseinheiten umfasst - war in der Ukraine nicht zu bewerkstelligen. Leonid Kučma griff hier auf eine andere Strategie zurück: Durch wechselnde Koalitionen aus regionalen Unternehmerparteien, inszenierten Parteiprojekten der Präsidialadministration und personalistischen Parteien wurde

49 Vgl. ebenda, S. $564 \mathrm{ff}$.

50 Vgl. Joel C. Moses, Russian Local Politics in the Putin - Medvedev Era, in: Europe-Asia Studies, 62. Jg. (2010), H. 9, S. 1427 - 1452; J. Paul Goode, The Puzzle of Putin's Gubernatorial Appointments, in: Europe-Asia Studies, 59. Jg. (2007), H. 3, 365 - 399. 
die interne institutionelle Geschlossenheit der Verchovna Rada gezielt geschwächt. Auch Amtsnachfolger Viktor Jǔčcenko misslang der Versuch, eine stabile parlamentarische „Hausmacht “ in der Verchovna Rada zu errichten. ${ }^{51}$ Inzwischen ist in der Ukraine der erneute Übergang des Wahlsystems von geschlossenen Parteilisten zu einem Mischsystem beschlossen worden, was künftig eine Vertretung unabhängiger Abgeordneter mit starker regionaler Bindung begünstigen dürfte. ${ }^{52}$ Paradigmatisch lassen sich die substanziellen Unterschiede mit Hinblick auf die politische Repräsentation und den Parteienpluralismus in beiden Staaten als „dominante Machtpolitik“ in Russland gegenüber einem „schwachen, dysfunktionalen Pluralismus" in der Ukraine darstellen. ${ }^{53}$

\subsection{Fragmentierung und Desorganisation („fragmentation“)}

Welche Auswirkungen haben diese institutionellen Rahmenbedingungen auf das Abgeordnetenprofil, das Amtsverständnis der Parlamentarier und die Erfüllung von Parlamentsfunktionen? Zunächst unterscheiden sich die Karrierewege und sozialen Profile russischer und ukrainischer Abgeordneter deutlich von denen ihrer westeuropäischen Kollegen. Für beide Länder lässt sich eine Praxis der Elitenrekrutierung konstatieren, die sich kaum nach programmatisch-inhaltlichen Gesichtspunkten richtet. In der Verchovna Rada stellen noch immer politische Quereinsteiger mit schwacher parteipolitischer Anbindung einen Großteil der Abgeordneten. Seit der Übernahme des Präsidentenamtes durch Viktor Janukovyč im Februar 2010 nehmen jedoch der Organisationsgrad und der Einfluss der „Partei der Regionen“" in der Verchovna Rada kontinuierlich zu. Die Loyalität ukrainischer Parlamentarier gegenüber Parteien und Fraktionen bleibt dennoch außerordentlich schwach. Dies lässt sich zumindest teilweise aus dem beruflichen und sozialen Hintergrund der Abgeordneten erklären. Etwa zwei Drittel der ukrainischen Parlamentarier sind Geschäftsleute. Dies ist ein Trend, der sich auch in Vertretungskörperschaften auf der regionalen und lokalen Ebene fortsetzt. ${ }^{54}$ Die Vereinnahmung der Legislativorgane durch Abgeordnete aus der Wirtschaft hat dabei verheerende Folgen für das Parlament als Institution und die Erfüllung elementarer Parlamentsfunktionen. Zahlreiche Abgeordnete betreiben ihr Mandat de facto auf „Teilzeitbasis“. Bei ukrainischen Parlamentswahlen wird von einer - allerdings schwer nachweisbaren - weiten Verbreitung des Kaufs von Parteilistenplätzen ausgegangen. ${ }^{55}$

Ein weiteres Phänomen stellt der „politische Tourismus“ dar. Parteien werden demnach lediglich als Vehikel zur Erlangung des Mandats betrachtet, wobei Abgeordnete nach dem Einzug ins Parlament häufig mehrfach ihre Fraktionsangehörigkeit wechseln. Dies führt

51 Vgl. Ingmar Bredies, a.a.O. (Fn. 44), S. 155 - 164.

52 Vgl. Volodymyr Kurennyi, Zmišana systema uyboriv: upered, u mynule, 17. November 2011, http://www.pravda.com.ua/articles/2011/11/17/6760394/ (Abruf am 24. November 2011).

53 Vladimir Gelman, From 'Feckless Pluralism' to 'Dominant Power Politics'? The Transformation of Russia's Party System, in: Democratization, 13. Jg. (2006), H. 4, S. 545 - 561, S. 548 ff.

54 Vgl. Ingmar Bredies, a.a.O. (Fn. 44), S. 226 - 239.

55 Bei den ukrainischen Parlamentswahlen 2002 wurde ein Listenplatz unter den ersten zehn Kandidaten einer Partei oder eines Wahlblocks auf etwa 500.000 US-Dollar geschätzt. Vgl. Rosaria Puglisi, The Rise of the Ukrainian Oligarchs, in: Democratization, 10. Jg. (2003), H. 3, S. 99 123. 
zwangsläufig zu einer Verzerrung des Wählerwillens, da sich die Stimmenanteile gewählter Parteien nur bedingt in der realen Fraktionsgröße im Parlament niederschlagen. ${ }^{56}$ Dieser Umstand veranlasste den damaligen Präsidenten Viktor Juščnk $k o$ im April 2007 zur - formal verfassungswidrigen - Auflösung der Verchovna Rada, die vom Verfassungsgericht autorisiert wurde. Es wird davon ausgegangen, dass im Rahmen der „Monetarisierung“ parlamentarischer Prozesse und Strukturen die Entlohnung von Fraktionswechseln oder Abstimmungsverhalten eine gängige Praxis darstellt. ${ }^{57}$ Das Amtsverständnis der Parlamentarier ist in beiden Staaten durch den Repräsentationsstil eines imperativen Mandats geprägt. Die stärksten Loyalitätsbezüge bestehen in erster Linie zu finanz-industriellen Interessengruppen. Während in Russland jedoch - bedingt durch die herausragende Stellung von staatlichen Unternehmen und einen rigiden Wirtschaftsinterventionismus - die Vereinigung dieser Interessengruppen unter dem „Dach“ der regierungstreuen Partei „Einiges Russland“ weitestgehend gelungen ist, wird die Konfiguration in der Ukraine durch eine hoch fragmentierte und inkonsistente Pluralität geprägt. ${ }^{58}$

Diese Entwicklungen dürften der wesentliche Grund für das geringe Vertrauen der ukrainischen Bevölkerung in ihr Parlament sein. „Volle Unterstützung“ für die Verchovna Rada erklärten in Umfragen unter fünf Prozent der Bürger. Hingegen ergaben sich 2010/2011 für die russische Staatsduma Zustimmungswerte von immerhin 25 bis 30 Prozent. ${ }^{59}$ Es ist davon auszugehen, dass dieser vergleichsweise hohe Wert nicht ihrer institutionellen Leistung zuzuschreiben ist. Vielmehr scheint die von der präsidentennahen Partei „Einiges Russland“ dominierte Staatsduma als Institution von den hohen Zustimmungswerten für Premierminister Putin zu profitieren, gerade weil sie kein signifikantes Gegengewicht zur Exekutive darstellt. In der Staatsduma sind neben Unternehmern Staatsbeamte aus Militär, Sicherheits- und Geheimdiensten sowie die Gruppe von bekannten Persönlichkeiten aus Sport, Kultur und der Unterhaltungsbranche („,elebrities“) dominant. ${ }^{60}$

Gerade die offenen wie verborgenen Geschäftsinteressen zahlreicher Abgeordneter liefern sowohl in Russland als auch in der Ukraine einen kaum zu überschätzenden Einflusshebel zur „Domestizierung“ der Parlamente. Insbesondere die Abgeordnetenimmunität bietet dabei einen besonderen Anreiz, einen Sitz im Parlament anzustreben. Sie stellt für „Business-Abgeordnete“ eine Absicherungsstrategie ihrer Geschäftsinteressen dar, da dieses Privileg häufig als „Generalvollmacht" gegen jegliche Art von Strafverfolgung missverstanden wird. ${ }^{61}$ Darüber hinaus ermöglicht das Parlamentsmandat, Lobbyarbeit im Sinne eigener wirtschaftlicher Interessen zu betreiben.

56 Vgl. Sarah Whitmore, State-building in Ukraine: The Ukrainian Parliament 1990-2003, London 2004, S. $81 \mathrm{ff}$.

57 Vgl. Ingmar Bredies, a.a.O. (Fn. 44), S. $181 \mathrm{ff}$.

58 Vgl. Heiko Pleines, Ukrainische Seilschaften. Informelle Einflussnahme in der ukrainischen Wirtschaftspolitik 1992-2004, Münster 2005; Vladimir Shlapentokh, Big Money as an Obstacle to Democracy in Russia, in: Journal of Communist Studies and Transition Politics, 24. Jg. (2008), H. 4, S. $512-530$, S. $521 \mathrm{ff}$.

59 http://www.uceps.org/ukr/poll.php?poll.id=68; http://bd.fom.ru/pdf/d07div11.pdf (Abruf am 24. November 2011).

60 Vgl. Eugene Huskey, a.a.O. (Fn. 19), S. 364 ff.; Elena Semenova, How to Become an MP: Die Rekrutierung von deutschen und russischen Parlamentariern im Vergleich (SFB 580, Mitteilungen H. 28/2008), S. $60 \mathrm{ff}$.

61 Vgl. Ingmar Bredies, Über dem Gesetz: Das „andere“ Amtsverständnis ukrainischer Parlamentarier und die Frage der Abgeordnetenimmunität, in: Ukraine-Analysen, (2009), H. 55, S. 2 - 5. 
Die „Entprofessionalisierung“ des Abgeordnetenmandats trägt somit maßgeblich zur Zerstörung parlamentarischer Deutungsmacht bei. Dabei liefert die zweifelhafte Reputation von Parlamentariern und Legislativorganen in Russland und der Ukraine der Exekutive auch einen Vorwand zur institutionellen Einhegung. So wurde durch die Präsidenten ganz bewusst ein Image des Parlaments als ineffiziente Institution mit unprofessionellen, lediglich um ihr Eigenwohl besorgten Abgeordneten kultiviert. Viktor Juščenko verkündete im Vorfeld der vorgezogenen Parlamentswahl 2007: „Die Volksabgeordneten sollen im Parlament neue Gesetze verabschieden und sich nicht dort vor ihnen verstecken."62 Bei eben dieser Wahl traten dann auch alle relevanten Parteien selbst im Wahlkampf für die Abschaffung der Abgeordnetenimmunität - und somit die Demontage ihrer eigenen Position - ein, ohne jedoch nach der Wahl dieses Vorhaben in die Praxis umzusetzen. Obwohl der Verchovna Rada wesentlich mehr Spielraum zur Ausgestaltung und Erfüllung von Parlamentsfunktionen verblieben ist als der Staatsduma, konnte das Parlament als Institution davon nicht profitieren.

\section{Schlussfolgerungen: Bedingungsfaktoren intitutioneller Einhegung}

Vor allem die Präsidenten haben großen Erfindungsreichtum bei der Zerstörung von Parlamentsmacht in Russland und der Ukraine demonstriert. Diese „Domestizierung “ umfasst den formal wie informal erwirkten Verlust institutioneller Zuständigkeiten und Befugnisse des höchsten Legislativorgans („disempowerment“), die Regulierung des Zugangs zu Parlamentsmandaten und Beeinflussung der personellen Zusammensetzung von Parlamenten (,agent control“) sowie die Schwächung der parlamentarischen Handlungskapazität durch gezielte Desorganisation („fragmentation“).

Sowohl in Russland als auch in der Ukraine manifestiert sich hierin die Einschränkung des politischen Pluralismus, was eine Bestimmung des Regimetypus in beiden Ländern als Autoritarismus rechtfertigt. In Russland lässt sich gegenwärtig kaum von einer institutionellen Autonomie der Staatsduma sprechen, auch wenn die jüngste Parlamentswahl vom 4. Dezember 2011 der Partei der Macht „Einiges Russland“ empfindliche Stimmenverluste einbrachte. Unter diesen Gegebenheiten können elementare Parlamentsfunktionen nicht erbracht werden. Gerade auch nach der Präsidentschaftswahl vom 4. März 2012 und der erneuten Übernahme des Präsidentenamtes durch Vladimir Putin sind zumindest mittelfristig keine substantiellen Veränderungen zu erwarten. Die Ukraine zeichnet hingegen eine spezifische Pluralität des politischen Systems aus, die dennoch scharf von einem voll ausgeprägten Pluralismus rechtsstaatlicher Demokratien abzugrenzen ist. Diese beschränkte Pluralisierung lässt sich als „pluralism by defaul “ ${ }^{63}$ darstellen. Demnach ist die Öffnung des politischen Systems weniger die Folge einer originären, zielgerichteten Demokratisierung als vielmehr ein unintendiertes Nebenprodukt eines gescheiterten Autoritarismus, in dem die Monopolisierung politischer Entscheidungsprozesse misslungen ist. Dieser Umstand hat stets eine gewisse Autonomie der Verchovna Rada gegenüber der Exekutive si-

62 Ebenda, S. 5.

63 Lucan A. Way, Authoritarian State-Building and the Sources of Regime Competitiveness in the Fourth Wave: The Cases of Belarus, Moldova, Russia, and Ukraine, in: World Politics, 57. Jg. (2005), H. 2, S. $231-261$, S. $232 \mathrm{ff}$. 
chergestellt. Der regionalen Heterogenität des Landes, die von Politikwissenschaftlern noch immer überwiegend als problematisches und konfliktträchtiges Entwicklungshemmnis aufgefasst wird, ist zu verdanken, dass jeglicher Zentralisierung und Machtkonzentration Grenzen gesetzt sind. Hinzu kommen im Vergleich zu Russland weitere strukturelle „Vorteile" - so die Schwäche staatlicher Institutionen und eine Volkswirtschaft, die sich nicht auf die Einnahmen von schier unerschöpflichen Ressourcenvorkommen und deren gezielte Allokation stützen kann. ${ }^{64}$ Wenn auch die institutionelle Konfiguration in Russland und der Ukraine unterschiedlich ausgeprägt ist, so haben sich dennoch weder die Staatsduma noch die Verchovna Rada als Garanten einer nachhaltigen politischen und gesellschaftlichen Demokratisierung erwiesen.

Abschließend sei ausdrücklich darauf hingewiesen, dass die institutionelle Einhegung osteuropäischer Parlamente grundsätzlich anderer Natur ist als die Tendenzen der Entparlamentarisierung, die von Politikwissenschaftlern in Hinblick auf westliche Regierungssysteme seit einiger Zeit diskutiert werden. Insofern es hier zu einer Schwächung realer politischer Gestaltungsräume und Mitwirkungspotenziale nationaler und subnationaler Parlamente gekommen ist, ist diese vorrangig das Resultat der Europäisierung und Internationalisierung politischer Entscheidungsprozesse mit ihrer spezifischen Exekutivdominanz und oft prekären demokratischen Legitimierung. ${ }^{65}$

64 Vgl. Paul D’Anieri, Structural Constraints in Ukrainian Politics, in: East European Politics and Societies, 25 Jg. (2011), H. 1, S. 28 - 46; Taras Kuzio, Political Culture and Democracy. Ukraine as an Immobile State, in: European Politics and Societies, 25. Jg. (2011), H. 1, S. 88 - 113.

65 Vgl. Suzanne S. Schüttemeyer, Modewort oder Alarmsignal? Befunde und Überlegungen zur Entparlamentarisierung, in: Werner J. Patzelt / Martin Sebaldt / Uwe Kranenpohl (Hrsg.), Res publica semper reformanda. Wissenschaft und politische Bildung im Dienste des Gemeinwohls, Wiesbaden 2007, S. 240 - 253, S. $242 \mathrm{ff}$. 